\title{
STUDY OF FOSFOMYCIN TROMETAMOL IN ACUTE LOWER URINARY TRACT INFECTIONS
}

\author{
Preetkamal Bedi' ${ }^{1}$ Kanwardeep Singh ${ }^{2}$, Prabjot Wariach ${ }^{3}$
}

1 Professor, Department of Obstetrics and Gynaecology, Government Medical College, Amritsar.

${ }^{2}$ Associate Professor, Department of Microbiology, Government Medical College, Amritsar.

${ }^{3}$ Resident, Department of Obstetrics and Gynaecology, Government Medical College, Amritsar.

ABSTRACT
OBJECTIVES
To evaluate the efficacy of single oral dose of Fosfomycin Trometamol (FT) in patients of uncomplicated lower urinary tract
infections.

\section{METHODS}

One hundred women between 18-65 years' age group with uncomplicated Urinary Tract Infection (UTI) with culture positive for E. coli and Enterococcus were enrolled in this prospective study. Patients with culture positive for E. coli and Enterococcus and with in-vitro susceptibility to Fosfomycin Trometamol (FT) as tested by Kirby-Bauer disc diffusion method were given single oral dose of Fosfomycin trometamol, i.e. 3 g. These patients were followed up for clinical and bacteriological cure and any adverse effects.

\section{RESULTS}

The incidence of E. coli infection was $86 \%$ and Enterococcus infection was 14\%; 78\% of E. coli isolates were susceptible to FT with $22 \%$ being resistant; $86 \%$ of Enterococcus isolates were susceptible to FT with $14 \%$ being resistant. Incidence of adverse effects of FT was only $2 \%$.

\section{CONCLUSIONS}

Fosfomycin trometamol might be considered as a promising single dose oral antibiotic for uncomplicated UTI due to E. coli and Enterococcus spp.

\section{KEYWORDS}

Urinary Tract Infections, Fosfomycin Trometamol, E. coli, Enterococcus, Adverse Effects.

HOW TO CITE THIS ARTICLE: Bedi P, Singh K, Wariach P. Study of fosfomycin trometamol in acute lower urinary tract infections. J. Evolution Med. Dent. Sci. 2016;5(31):1649-1650, DOI: 10.14260/jemds/2016/388

\section{INTRODUCTION}

Urinary tract infection is the second most common bacterial infection after respiratory tract infection. These infections occur more frequently in female population, almost four times than males with $60 \%$ of women having at least one infection at some point in their lives. They occur most frequently in sexually active females with $10 \%$ of women getting infection every year. Recurrences are common with nearly half of people getting a second infection within a year, either with different organism or reinfection with the same organism. Risk factors include female anatomy, sexual intercourse, family history or it may also result from blood borne infection. Uncomplicated UTI is defined as an infection occurring in a structurally and functionally normal urinary tract. Complicated UTI refers to infection in a urinary tract with abnormalities. The pathogenesis of UTI depends on the results of an interaction between bacterial virulence and host defence mechanism.

Although UTIs are caused by many micro-organisms, the most frequent aetiological agent is E. coli ( $80 \%$ of cases). ${ }^{1}$

Financial or Other, Competing Interest: None.

Submission 29-02-2016, Peer Review 24-03-2016,

Acceptance 30-03-2016, Published 18-04-2016.

Corresponding Author:

Dr. Kanwardeep Singh,

Associate Professor,

Department of Microbiology,

Government Medical College,

Amritsar.

E-mail: kdmicrogmcasr@gmail.com

DOI: $10.14260 /$ jemds $/ 2016 / 388$
Uncomplicated infections can be diagnosed and treated based on symptoms alone. Oral antibiotics such as trimethoprim/sulfamethoxazole, nitrofurantoin or fosfomycin are typically first line antibiotics. Extended-spectrum Beta Lactamase (ESBL) produced by E. coli limits the treatment options. FT is a preferred broad-spectrum phosphoenolpyruvate analog antimicrobial agent in uncomplicated lower UTIs due to advantage of single dose use, rare side effects and low resistance rates. ${ }^{2}$

\section{MATERIALS AND METHODS}

This prospective study was conducted in the Department of Obstetrics and Gynaecology, Bebe Nanaki Hospital and Department of Microbiology, Govt. Medical College, Amritsar. Patients in age group of 18-65 years with complaints of dysuria, urgency, frequency, pain lower abdomen, fever, etc. were subjected to detailed history, clinical examination, urine microscopic examination and urine for culture sensitivity. Patients with structural abnormalities of urinary tract, complicating factors like diabetes mellitus, obstructive uropathy like ureteric stones were not included in the study. One hundred patients were selected with urine culture positive for E. coli and Enterococcus.

Midstream clean catch urine samples from patients were collected in sterile containers and transported to microbiology laboratory.

Isolation and identification of isolates from urine specimen was carried out by using a semi-quantitative culture method as per standard protocol. 
Antimicrobial-susceptibility testing was carried out using the Kirby-Bauer disc diffusion technique; $200 \mu \mathrm{g}$ fosfomycin (With $50 \mu \mathrm{g}$ glucose-6-phosphate) discs were used. The diameter of the zones of inhibition for fosfomycin were interpreted according to the CLSI guidelines [Susceptible ( $\geq 16 \mathrm{~mm})$, intermediate $(13-15 \mathrm{~mm})$ or resistant $(\leq 12 \mathrm{~mm})]$.

The patients susceptible to FT on urine for culture sensitivity test were given single oral dose of $3 \mathrm{~g}$ of FT stat. These patients were subjected to repeat urine culture sensitivity after 48 hours of drug intake. The results were tabulated and evaluated to find the efficacy of FT single dose in improving the clinical and bacteriological results.

\section{RESULTS}

In the present study, 78 out of $100(78 \%)$ patients were in age group of $18-30$ years and $20 \%$ of the patients belonged to age group of 31-45 years; 46-65 years of age group was represented by only $2 \%$ of patients. Out of 100 patients, $86 \%$ of the patients were found to be infected with E. coli and $14 \%$ of the patients were infected with Enterococci as per their urine for culture sensitivity report.

Out of 86 E. coli isolates, only 67 (78\%) were susceptible to FT and 19 (22\%) were resistant. Out of 14 Enterococcus isolates, 12 (86\%) were sensitive to FT and 2 (14\%) were resistant. The resistance to FT seems to be either chromosomally or plasmid mediated. The chromosomal mutations act by altering the intracellular transport mechanisms of fosfomycin. Fosfomycin may also become inactive because of mutations, which make its target enzyme (Pyruvyl transferase) unable to distinguish between the substrate (Phosphoenolpyruvic acid) and fosfomycin.

Out of 79 patients who were treated with $3 \mathrm{gm}$ single oral dose of FT, none of the patients showed persistence of infectious agent in the urine as evidenced by no growth of the offending organism in the repeat urine for culture sensitivity test.

Only $2 \%$ of the patients who were treated with FT showed adverse drug effects in the form of nausea and epigastric discomfort.

\section{DISCUSSION}

Uncomplicated urinary tract infection is an infection occurring in a structurally and functionally normal urinary tract. E. coli and Enterococcus spp. together account for more than $80 \%$ cases of uncomplicated UTIs. Sahni et al. and Mengeloglu et al. found the incidence of E. coli infection to be $77 \%$ and $78 \%$ respectively with incidence of Enterococcus to be around 9\%.3,4 7-10 days of antibiotic therapy is routinely recommended for such patients. However, it has become apparent that most women have only a superficial mucosal infection and can be cured with much shorter courses of therapy. Fosfomycin trometamol is a phosphonic acid derivative, which acts by inhibiting biosynthesis of peptidoglycans required for bacterial cell wall synthesis. It is given as a single oral dose of $3 \mathrm{~g}$.
In the present study, the efficacy of fosfomycin trometamol as a single dose oral treatment for achieving bacteriological cure ranged from $80-90 \%$. Raz et al. also found that FT was the most active antibiotic agent against $\mathrm{E}$. coli and Enterococcus spp. with an overall susceptibility of $83 \%$ and $99 \%$ respectively. 5

All the patients who were treated with FT in the present study showed complete clinical and bacteriological cure. Neumann et al. also found that clinical and bacteriological cure was achieved in 17 out of 18 patients (94\%) with UTI. ${ }^{6}$ Single dose oral treatment offered $100 \%$ compliance as observed in the present study. In overall evaluation, the most frequently observed adverse events were diarrhoea, nausea, epigastric/abdominal pain and headache. Naber et al. also demonstrated that this drug is generally safe and well tolerated. ${ }^{7}$

\section{CONCLUSIONS}

The results of the present study indicate the patients in the study group had better outcomes in terms of improvement of symptoms of acute lower UTI and bacteriological cure with minimum incidence of adverse drug effects. FT offers better patient compliance on the account of single oral dose. Thus fosfomycin trometamol might be considered as a promising oral antibiotic for uncomplicated urinary tract infections due to E. coli isolates and Enterococcus spp.

\section{REFERENCES}

1. Kahlmeter G. The ECO SENS project: a prospective, multinational, multicenter epidemiological survey of the prevalence and antimicrobial susceptibility of urinary tract pathogens-interim report. J Antimicrob Chemother 2000;46(1):15-22. discussion 63-5.

2. Eschenburg S, Priestman M, Schonbrunn E. Evidence that the fosfomycin target cys 115 in UDP-Nacetylglucosamine enolpyruvyl transferase (MurA) is essential for product release. J Biol Chem 2005;280(5):3757-63.

3. Sahni RD, Balaji V, Varghese R, et al. Evaluation of fosfomycin activity against uropathogens in a fosfomycinnaïve population in South India: a prospective study. Future Microbial 2013;8(5):675-80.

4. Mengeloglu FZ, Demircan F, Oduncu MK. Evaluation of in vitro susceptibles of escherichia coli strains isolated from urine cultures to fosfomycin. Ankem Derg 2011;25:99102.

5. Raz R. Fosfomycin: an old-new antibiotic. Clin Microbial Infect 2012;18(1):4-7.

6. Neuman M, Rufin F. Activity of monuril in lower urinary tract infections due to fosfomycin-resistant bacteria. Eur Urol 1987;13(1):105-7.

7. Naber KG, Johnson FN. The safety and tolerability of fosfomycin trometamol. Rev Contemp Pharmacother 1995;6:63-70. 